\title{
Average characteristics of the midtail plasma sheet in different dynamic regimes of the magnetosphere
}

\author{
N. P. Dmitrieva, V. A. Sergeev, and M. A. Shukhtina \\ V. A. Fock Institute of Physics, St.-Petersburg State University, St.-Petersburg, Russia \\ Received: 8 August 2003 - Revised: 26 January 2004 - Accepted: 11 February 2004 - Published: 14 June 2004
}

\begin{abstract}
We study average characteristics of plasma sheet convection in the middle tail during different magnetospheric states (Steady Magnetospheric Convection, SMC, and substorms) using simultaneous magnetotail (Geotail, $15-35 \mathrm{R}_{E}$ downtail) and solar wind (Wind spacecraft) observations during 3.5 years. (1) A large data set allowed us to obtain the average values of the plasma sheet magnetic flux transfer rate $\left(\mathrm{E}_{y}\right)$ and directly compare it with the dayside transfer rate $\left(\mathrm{E}_{\mathrm{mod}}\right)$ for different magnetospheric states. The results confirm the magnetic flux imbalance model suggested by Russell and McPherron (1973), namely: during SMC periods the day-to-night flux transport rate equals the global Earthward plasma sheet convection; during the substorm growth phase the plasma sheet convection is suppressed on the average by $40 \%$, whereas during the substorm expansion phase it twice exceeds the day-to-night global flux transfer rate. (2) Different types of substorms were revealed. About 1/3 of all substorms considered displayed very weak growth in the tail lobe magnetic field before the onset. For these events the plasma sheet transport was found to be in a balance with the day-to-night flux transfer, as in the SMC events. However, the lobe magnetic field value in these cases was as large as that in the substorms with a classic growth phase just before the onset (both values exceed the average level of the lobe field during the SMC). Also, in both groups similar configurational changes (magnetic field stretching and plasma sheet thinning) were observed before the substorm onset. (3) Superimposed epoch analysis showed that the plasma sheet during the late substorm recovery phase has the characteristics similar to those found during SMC events, the SMC could be a natural magnetospheric state following the substorm.
\end{abstract}

Key words. Magnetospheric physics (Solar windmagnetosphere interactions; magnetotail; storms and substorms)

Correspondence to: N. P. Dmitrieva

(dmitrieva@geo.phys.spbu.ru)

\section{Introduction}

Magnetospheric convection is the basic phenomenon, which determines the strength and consequences of the externallydriven disturbances in the magnetosphere. Meanwhile, the enhanced energy supply from the solar wind to the magnetosphere during time periods of southward IMF may lead to very different dynamical regimes, namely, the substorms or Steady Magnetospheric Convection (SMC). The difference is believed to appear due to the specific difference between the two segments of the global circulation pattern. The magnetic flux transport from the dayside merging region to the magnetotail (DT segment) is characterized by a powerful solar wind driver and by strong $\mathrm{B}$ (low plasma $\beta$ ) conditions in the magnetospheric part of the transported flux tubes. The return convection from the tail reconnection region to the dayside magnetopause (TD segment) has different properties. It has to pass through the high $\beta$ plasma sheet where the pressure gradients building up during convection may modify (even stop or locally reverse) the return flow (Erickson, 1992). This can temporarily lead to the imbalance between DT and TD flux transport rates. The global magnetic flux transport rate for the DT segment can be computed in some cross section of the magnetotail as $\Delta \Phi_{B}=\int \mathrm{B}_{n} \mathrm{~V}_{s w}$ ds (integrating from dawn to dusk along the magnetopause, where $\mathrm{B}_{n}$ is the normal magnetic field component at the magnetopause and $\mathrm{V}_{S W}$ is the magnetosheath flow velocity, nearly equal to the solar wind velocity). The $\Delta \Phi_{B}$ value from the strong-B region near the magnetopause is expected to be mapped to the ionosphere, where it can be observed as the potential drop across the polar cap, $\Phi_{P C}$. This quantity is possible to measure with the radars and spacecraft instruments. In the TD segment a similar estimate can be done by integrating in the plasma sheet between the dawn and dusk points at the magnetopause: $\Delta \Phi_{P S}=\int(\boldsymbol{V} \times \boldsymbol{B})_{y} \mathrm{dy}$, where $\boldsymbol{V}$ and $\boldsymbol{B}$ are measured in the plasma sheet. In the high- $\beta$ plasma sheet the motion of equatorial part of the flux tube is not automatically mapped into the ionosphere, therefore, in situ measurements are required to control $\Delta \Phi_{P S}$. If $\Delta \Phi_{B}$ and $\Delta \Phi_{P S}$ 
Table 1. Comparison of the modeled and measured plasma sheet electric field values for SMC state.

\begin{tabular}{lccc}
\hline References & Potential drop $\left(\Delta \Phi_{\text {mod }}\right)$ & $<\mathrm{E}_{\text {mod }}>=\Delta \Phi_{\text {mod }} / 2 \mathrm{R}_{\text {mod }}$ & $\mathrm{K}=<\mathrm{E}_{y}>/<\mathrm{E}_{\text {mod }}>$ \\
\hline Boyle et al. (1997) & $\Delta \Phi=(\mathrm{v} / 100)^{2}+11.7 * \mathrm{~B} * \sin ^{3}(\theta / 2)$ & $0.19( \pm 0.10) \mathrm{mV} / \mathrm{m}$ & 1.21 \\
\hline Lu et al. (1989) & $\Delta \Phi=12.7+0.041 * \mathrm{v} * \mathrm{~B} * \sin ^{3}(\theta / 2)$ & $0.23( \pm 0.13) \mathrm{mV} / \mathrm{m}$ & 1.00 \\
\hline Reiff et al. (1981) & $\Delta \Phi=30+0.0061 * \mathrm{v} * \mathrm{~B}{ }^{2} * \sin ^{4}(\theta / 2)$ & $0.25( \pm 0.12) \mathrm{mV} / \mathrm{m}$ & 0.92 \\
\hline
\end{tabular}

are evaluated in the same tail cross-section, they should balance each other in the steady state known as Steady Magnetospheric Convection (SMC). Otherwise, according to the Faraday law the tail magnetic flux $\mathrm{F}_{T}$ computed in the half of tail cross section will change with time as

$d F_{T} / d t=\Delta \Phi_{B}-\Delta \Phi_{P S}$

The character of changes should depend on the kind of imbalance in Equation (1). The enhanced dayside reconnection (large $\Delta \Phi_{B}$ ) but depressed plasma sheet convection $\left(\Delta \Phi_{P S} \ll \Delta \Phi_{B}\right)$ would result in the magnetic flux storage in the tail (substorm growth phase), whereas in the opposite case, $\Delta \Phi_{P S} \gg \Delta \Phi_{B}$, one has the fast flux unloading (substorm expansion phase). This explanation of substorms has been outlined a long time ago by Russell and McPherron (1973). Since then, it has been illustrated in many case studies (e.g. Lyons et al., 2001) for recent examples), as well as in 3D-MHD simulations (e.g. Raeder et al., 2001) and has become a part of commonly accepted substorm paradigm.

There are still two kinds of problems/uncertainties with this explanation. The first one is that very little quantitative work has been reported so far to check the transport balance (1). There are only two papers that we know of whose authors statistically investigated the magnetic flux imbalance rate $\mathrm{dF}_{T} / \mathrm{dt}$ as a function of the solar wind parameters and (based on that) who discussed its relationship with the dayside merging rate $\left(\Delta \Phi_{B}\right)$. Both studies, Holzer and Slavin (1979), who evaluated dayside magnetic flux erosion, and Rybal'chenko and Sergeev (1985), who analysed the magnetic flux increase in the tail during the substorm growth phase, concluded that $\mathrm{dF}_{T} / \mathrm{dt}$ values are proportional (and roughly equal to) $\Delta \Phi_{B}$, although $\Delta \Phi_{P S}$ was not actually controlled in these analyses. An important case study of a few substorms have been published by Nakamura et al. (1999), who compared the plasma sheet convection as measured by the Geotail spacecraft with the dayside merging rate (based on $\Phi_{P C}$ ). Although their results provided a nice qualitative illustration of the above-mentioned magnetic flux imbalance paradigm, they also displayed a principal difficulty of such comparisons: the very bursty (spatially and temporally localized) character of the plasma sheet convection, where the electric field variability by an order of magnitude exceeds the average value, $\delta \mathrm{E} \gg \mathrm{E}_{A V E}$ (e.g. Angelopoulos et al., 1992). Therefore, in order to obtain representative estimates of $\Delta \Phi_{P S}$ required to make accurate comparisons between the different terms in Eq. (1), one has to do a gross averaging over space and time in many events. Such a statistical study is our first goal in this paper.

Another problem with the magnetic-flux-imbalance substorm paradigm is that its plasma mechanism is not established yet, and that is the reason why (and when) the magnetotail system selects the substorm or the SMC mode is unclear. Keeping in mind the possibility of these two alternatives, one should also ask if they represent well separated individual states or whether there exists a continuum of states between substorm and SMCs, that is a number of different dynamical paths for a system to behave under the similar solar wind conditions. A possible way to look at this problem is to obtain and compare the average plasma sheet parameters for different dynamical states of the tail distinguished by the amount of the flux imbalance $\mathrm{dF}_{T} / \mathrm{dt}$ (estimated from variations of the measured lobe magnetic field $\mathrm{B}_{T}$ ). Many examples of observations could be found in event studies published so far, but no gross-averaged values characterizing different states are available. Such average values will be obtained from this paper for substorms and for steady convection in the midtail region (15-35 $\left.R_{E}\right)$, including the values and behavior of plasma sheet convection, magnetic field $\mathrm{B}_{z}$-component and plasma $\beta$ parameter (characterizing the variations of plasma sheet thickness) under the controlled solar wind electric field and RAM pressure. We also compare parameters for two different types of substorms and discuss the differences between substorms and SMCs from the viewpoint of the flux imbalance.

\section{Data analysis}

\subsection{Data base and event selection}

We take advantage of exploiting the extensive data base of the merged solar wind and plasma sheet observations classified by different dynamical states, previously used to obtain the statistical regression models for the lobe magnetic field and tail flaring angle (also the tail radius and magnetic flux) as a function of solar wind parameters (Shukhtina et al., 2004), thereafter referred to as Paper 1 . Referring to this paper for further details, we briefly review the data selection criteria.

We used Geotail plasma and magnetic field measurements and Wind solar wind data during the period January 
1995-April 1998 when Geotail was in the middle tail (at $\left.-35 R_{E}<\times<-15 R_{E},|\mathrm{Y}|<15 R_{E}\right)$ and data from both spacecraft were available. The condition $\mathrm{X}<-15 R_{E}$ was set to work in the region where the tail approximation (and resulting homogeneity of the tail lobe field), as well as the vertical pressure balance $\left(\mathrm{B}_{L}^{2} / 2 \mu_{0}=\mathrm{B}^{2} / 2 \mu_{0}+\mathrm{nkT}\right)$ are both valid. The midtail parameters were calculated based on the original 12-s Geotail magnetic field and ion plasma moments data and were finally averaged over $6 \mathrm{~min}$. These parameters included:

(1) The equivalent lobe magnetic field $\mathrm{B}_{L}$ computed from vertical pressure balance and reduced to the $\mathrm{r}=20 R_{E}$ distance (to correct for strong radial variation of the lobe field). The formula obtained from the empirical relationship published by Fairfield and Jones (1996) has been used for such a correction: $\mathrm{B}_{L}(20)=\mathrm{B}_{L}(\mathrm{r})$ $\left(7.47+1659.2 / \mathrm{r}^{1,46}\right) /\left(7.47+1659.2 / 20^{1.46}\right)$; (2) GSM B magnetic field component; (3) $\mathrm{V}_{x}$-component of plasma velocity; (4) Convection electric field $\mathrm{E}_{y}=(\boldsymbol{V} \times \boldsymbol{B})_{y}$ characterizing the local flux transport rate; (5) Local plasma beta $\beta=\mathrm{nkT} /\left(\mathrm{B}_{L}^{2} / 2 \mu_{0}\right)$.

Solar wind magnetic and plasma data from the WIND spacecraft with an $\sim 1$-min resolution available from CDAWeb were averaged over $6 \mathrm{~min}$. after being shifted in time to Geotail location according to $\Delta \mathrm{t}=\left(\mathrm{X}_{\text {Wind }}-\right.$ $\mathrm{X}_{\mathrm{Geotail}} / \mathrm{V}_{\mathrm{SW}}$. The computed parameters included: (1) solar wind dynamic pressure $\mathrm{P}_{d}=1.94 \mathrm{n}_{p} \mathrm{~V}_{\mathrm{sw}}^{2}$ (the coefficient 1.94 includes the contribution of $\alpha$-particles, see, e.g. (Tsyganenko, 1996).) and (2) the modeled cross-tail electric field $\mathrm{E}_{\text {mod }}=\Delta \Phi_{\bmod } / 2 R_{\text {mod }}$, where $\Delta \Phi_{\text {mod }}$ and $R_{\text {mod }}$ are the empirical values of the cross-polar cap potential drop and magnetotail radius calculated based on solar wind/IMF parameters (see Sect. 2.2). The 6-min averaging interval was taken as it gives the solar wind travel distance $\sim 20-30 R_{E}$ which is about the size of midtail magnetotail region. Ground indices (polar cap PC index from Thule, SYM and AE indices) and high-latitude magnetograms were also used to identify the SMC events and substorms.

The formal criteria to identify the events were as follows (see Shukhtina et al. for more details). Substorm onsets (onsets of the substorm expansion phase) were primarily identified based on the distinct signature of isolated strong unloading in the midtail ( $\mathrm{B}_{L}$ decrease, usually accompanied by sharp $\mathrm{B}_{Z}$ increase/decrease at Geotail). It was then checked that corresponding negative magnetic bays at the nightside auroral stations or in the AL index with amplitude $>100 \mathrm{nT}$ were also observed. By the name "isolated" we mean that the lobe field $\mathrm{B}_{L}$ was smoothly changing or stable after the previous substorm (this situation rather corresponding to the "main onset" by the definition given by Hsu and McPherron, 1998). Superposed analysis required the presence of continuous data during $2 \mathrm{~h}$ before substorm onset and $3 \mathrm{~h}$ after it. 145 events satisfying these criteria were selected. To identify the event as the steady magnetospheric convection (SMC) we required considerable external driving $\left(\mathrm{E}_{\bmod }>0.1 \mathrm{mV} / \mathrm{m}\right.$, corresponding to $\mathrm{B}_{z} \mathrm{IMF} \approx-1 \mathrm{nT}$ and $\mathrm{V}_{S W} \approx 450 \mathrm{~km} / \mathrm{s}$ with $\mathrm{PC}$ index following $\mathrm{E}_{\mathrm{mod}}$ variations) without substorms during

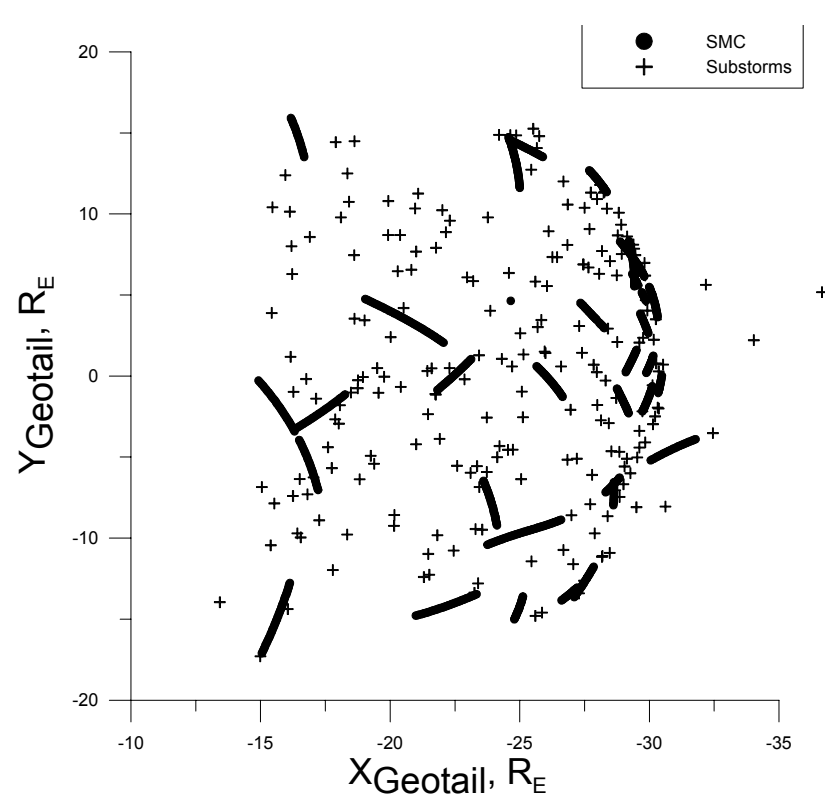

Fig. 1. Geotail position in GSM coordinates during SMCs (circles) and substorms onsets (crosses).

at least the preceding hour (both lobe field variations and $\mathrm{AE}$ index, as well as auroral zone magnetograms were checked). The lobe field variations were allowed if they followed the $\mathrm{B}_{L}$ variations due to solar wind $\mathrm{P}_{d}$ variations (within 5\% of prediction by statistical relationship from Fairfield and Jones, 1996). Only the SMC time periods longer than $3 \mathrm{~h}$ with $\mathrm{AE}>50 \mathrm{nT}$ were considered. As a result 32 such SMC events were identified with the total amount of 10856 min-averaged samples of observations.

Figure 1 presents the Geotail position during the time periods studied. It shows that the Geotail measurements cover uniformly the midtail region for substorms and SMCs, therefore the averaging over these events really provides a homogeneous averaging in space as required to obtain representative estimates of the global plasma sheet flux transport rate.

\subsection{Relationship between measured parameters and global transfer rates; Evaluation of the dayside flux transport rate}

The global return (nightside-to-dayside) flux transfer rate can be controlled based on $\mathrm{E}_{y}=(\mathrm{VxB})_{y}$ values measured in situ by Geotail spacecraft (giving the nightside flux transfer rate estimate $\Delta \Phi_{P S}=2 \mathrm{R}_{T} \mathrm{E}_{y}$, where $\mathrm{R}_{T}$ is the tail radius). The variations of the lobe magnetic flux $\mathrm{F}_{T}$ can be evaluated from the variations of the lobe magnetic field reconstructed from Geotail measurements as well (using half-circle approximation for the lobe, that is $\left.\mathrm{F}_{T}=\mathrm{B}_{L} \pi \mathrm{R}_{T}^{2} / 2\right)$. As mentioned in the Introduction, the DT flux transport rate can be estimated by the cross-polar cap potential drop $\Phi_{P C}$. In our case it is evaluated based on the solar wind data using previously found empirical relationships. We considered three statistical models of $\Delta \Phi_{\text {mod }}$ presented in Reiff et al. (1981), Lu et al. (1989) 

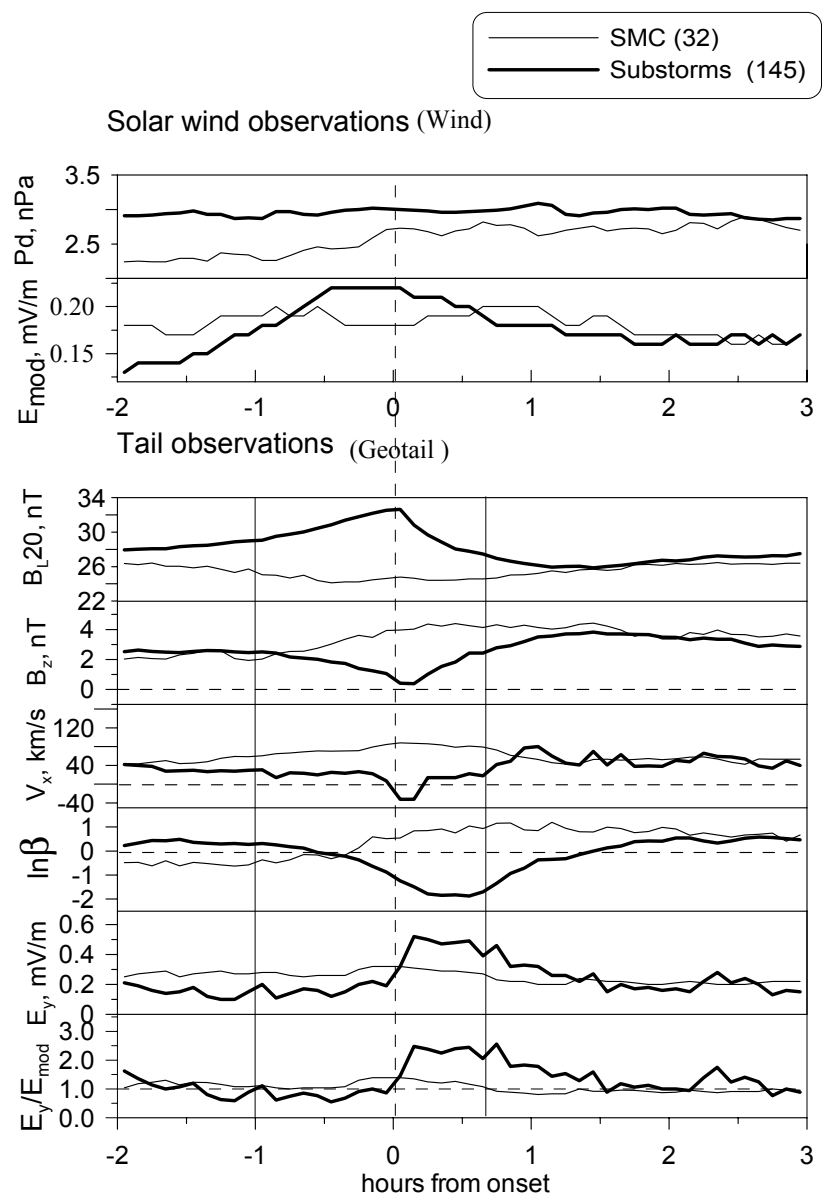

Fig. 2. Variations of the average values of the solar wind (top panel) and plasma sheet (bottom panel) parameters for SMC and substorm events. $\mathrm{E}_{\text {mod }}$ was calculated according to model number 1 (see Table). $\mathrm{V}_{x}$ and $\mathrm{E}_{y}$ were additionally time-averaged (with 1-h wide sliding window).

and Boyle et al. (1997) (see Table 1). The model cross-polar cap electric field was calculated as $E_{\text {mod }}=\Delta \Phi_{\text {mod }} / 2 R_{\text {mod }}$, where the $R_{\text {mod }}$ value was calculated using the model of Petrinec and Russel (1996) for average spacecraft position (at $\mathrm{X}=-25 R_{E}$ ).

During steady conditions the dayside and return flux transport rates should balance each other $\left(\Delta \Phi_{P S} \approx \Delta \Phi_{B}\right.$, which means $<$ Ey $>\approx \mathrm{E}_{\bmod }$, suggesting the spatially-averaged plasma sheet electric field to be $<\mathrm{Ey}>=\Delta \Phi_{P S} / 2 \mathrm{R}_{T}$ ), so to check the quality of our estimates we first compare the corresponding values averaged over all SMC events. It is worth noting that the large variability of plasma flows and electric field is common during the SMC (Baumjohann, 1993, Sergeev et al., 1996), so the instantaneously measured $\mathrm{E}_{y}$ strongly deviates from $\left\langle\mathrm{E}_{y}>\right.$ (which controls the flux balance). Therefore, we don't expect to obtain the $\mathrm{E}_{y}$ balanced until we average $\mathrm{E}_{y}$ over either space or time (during steady convection). For all 1085 individual 6-min samples available the average plasma sheet transport appeared to be $<\mathrm{E}_{y}>=0.23 \mathrm{mV} / \mathrm{m}$ (with the standard deviation $\delta=0.54$, twice larger than the mean value). The average dayside flux transport rate $\mathrm{E}_{\text {mod }}$, estimated based on solar wind parameters using different empirical potential models, is given in the Table. According to all three modes $<\mathrm{E}_{\bmod }>\approx<\mathrm{Ey}>$ with $20 \%$ - accuracy. This agreement assures us that plasma sheet measurements (after gross averaging) give quite representative values of the nightside flux transport. With this encouraging agreement we continue the data analysis.

\subsection{Superimposed epoch analysis; Comparison of steady convection and substorms}

The superimposed epoch analysis is a powerful method of averaging, whose strength was many times demonstrated in the application to substorms (e.g. Foster et al., 1971; Caan et al., 1978). Figure 2 presents the result from the 5-h long time interval $\left(\mathrm{T}_{0}-2 \mathrm{~h}, \mathrm{~T}_{0}+3 \mathrm{~h}\right)$. For substorms the choice of $\mathrm{T}_{0}$ is straightforward; it corresponds to the onset of a sharp lobe field $\left(\mathrm{B}_{L}\right)$ decrease. For $\mathrm{SMC}$ events $\mathrm{T}_{0}$ was taken at the beginning of the SMC period, defined according to SMC criteria (see Sect. 2.1). Unlike $\mathrm{P}_{d}, \mathrm{E}_{\text {mod }}$, Geotail $\mathrm{B}_{L}, \mathrm{~B}_{z}$ and $\beta$ traces, which demonstrate the stable behavior after averaging over $32 \mathrm{SMC}$ events, $\mathrm{V}_{x}$ and $\mathrm{E}_{y}$ at Geotail were still variable, indicating that this averaging was not sufficient to smooth the variability caused by the plasma sheet BBFs. Therefore, we applied the additional time averaging (with 1-h wide sliding window) of $<\mathrm{E}_{y}>$ for the SMC data set. After that (see Fig. 2) the $\left\langle\mathrm{E}_{y}>\right.$ appeared to be reasonably stable at the level about $0.2 \mathrm{mV} / \mathrm{m}$ during the $\mathrm{T}_{0}$ to $\mathrm{T}_{0}+3 \mathrm{~h}$ time interval, which means $<\mathrm{E}_{y}>/<\mathrm{E}_{\bmod }>\sim 1.0$, that is the flux transfer balance.

The average magnetotail parameters characterizing the SMC state are: $\quad<\mathrm{B}_{L}(20)>=26 \mathrm{nT}, \quad<\mathrm{B}_{z}>=4 \mathrm{nT}$, $<\ln \beta>=0.74,<\mathrm{E}_{y}>=0.23 \mathrm{mV} / \mathrm{m}$. This agrees with the previous case studies which showed that the midtail plasma sheet during SMC events is characterized by the thick plasma sheet, large closure of the magnetic flux through the plasma sheet and considerable earthward transport of the magnetic flux (e.g. Sergeev et al., 1996). The lobe magnetic field is smaller than its average value computed for the given solar wind conditions according to Fairfield and Jones (1996) which was emphasized in Paper 1.

The average midtail behavior during substorms demonstrates a number of previously known features, including three well-defined substorm phases, and tags them with average quantitative characteristics. The average growth phase begins $\sim 1 \mathrm{~h}$ before the substorm onset (in agreement with, e.g. Bargatze et al., 1985; Caan et al., 1978, etc.), in association with enhanced external energy input. The growth phase is characterized by $\mathrm{B}_{L}(20)$ growth by $4 \mathrm{nT}$, consistent with Rybal'chenko and Sergeev (1985) (13\% from the initial level), field line stretching $\left(\mathrm{B}_{z}\right.$ decrease combined with the $\mathrm{B}_{L}$ increase) and plasma sheet thinning (as manifested by $\beta$ decrease). The average plasma sheet flux transport rate during the growth phase, $\left\langle\mathrm{E}_{y}>=0.15 \mathrm{mV} / \mathrm{m}\right.$ value, is $35 \%$ depressed as compared to that during the SMC periods $(0.23 \mathrm{mV} / \mathrm{m})$ under the similar day-night transfer rate. This 
confirms in quantitative terms the association of the magnetic energy storage in the tail with an imbalance between the DT transport rate and return convection in the plasma sheet during the growth phase. Further details will be given later in Sect. 2.4.

The duration of the average substorm expansion phase is about $40 \mathrm{~min}$. The sharp $\mathrm{B}_{L}$ decrease (by 21\%) and $\mathrm{E}_{y}$ increase $\left(<\mathrm{E}_{y}>=0.48 \mathrm{mV} / \mathrm{m}\right), 2$ times larger as compared to the dayside transport rate or to that during the SMC state) manifest the strongly enhanced energy dissipation in the plasma sheet and reversed the flux transport imbalance $\left(\Delta \Phi_{P S} \gg \Delta \Phi_{\text {mod }} \sim \Delta \Phi_{B}\right)$. The $\mathrm{V}_{x}$ component demonstrates a bipolar (from tailward to earthward) variation accompanied by a bipolar $\mathrm{B}_{z}$ variation, with the earthward flow corresponding to $\mathrm{B}_{z}$ increase. This behavior is consistent with the near-Earth reconnection starting earthward of the spacecraft, but in 5-10 min. progressing further tailward (the mean Geotail position at the substorm onset is $\mathrm{X}=-27 R_{E}$ ). During the expansion phase the plasma sheet continues to be thin, its recovery (defined as a $\beta$ increase) on the average starts $\sim 40$ min after the onset. This time can be considered as the beginning of the recovery phase. The described time scales agree with the results by Baker et al. (1994), where the recovery phase was studied using superposed epoch analysis in the magnetotail, on the geostationary orbit and on the ground.

By the time of $\beta$-parameter recovery to its pre-substorm value (which occurred $\sim 40-50 \mathrm{~min}$. after the start of recovery phase) the other parameters $\left(\mathrm{B}_{L}, \mathrm{~B}_{z}, \mathrm{~V}_{x}, \mathrm{E}_{y}\right)$ also reach their saturation level. However, these new levels differ from those observed prior to the growth phase during the interval $\left(\mathrm{T}_{0}-1 \mathrm{~h} ; \mathrm{T}_{0}-2 \mathrm{~h}\right)$. Particularly, the $\mathrm{B}_{L}$ is reduced, whereas the $\mathrm{B}_{z}, \mathrm{E}_{y}$ and $\mathrm{V}_{x}$ values are enhanced as compared to those observed before the substorm, whereas the DT transport rate does not decrease after the end of expansion phase. As a result, in this data set of 145 substorms the average state of the midtail reached after the substorm approachs the state similar to that found in 32 steady convection events.

Thus, it seems that after the expansion phase the magnetotail recovers to the state with characteristics similar to those of the SMC state: stretched magnetic configuration and thin current sheet reappeared near the geosynchronous orbit (Pulkkinen et al., 1994), and thick current sheet with closed magnetic configuration are seen in the midtail, suggesting a kind of a hybrid state (Sergeev et al., 1994). In that sense the SMC regime looks like a natural magnetospheric state following the substorm, which is probably why all but one SMC period considered in our work was observed after substorms.

2.4 Substorms with/without the classic growth phase, role of the plasma sheet convection

Although the average substorm-related variations described in the previous section display the widely accepted portrait of the "classic substorm" in the magnetotail, the reality appears to be more complicated and variable. From all available 145 substorm events, the well-defined growth-phase-related BL
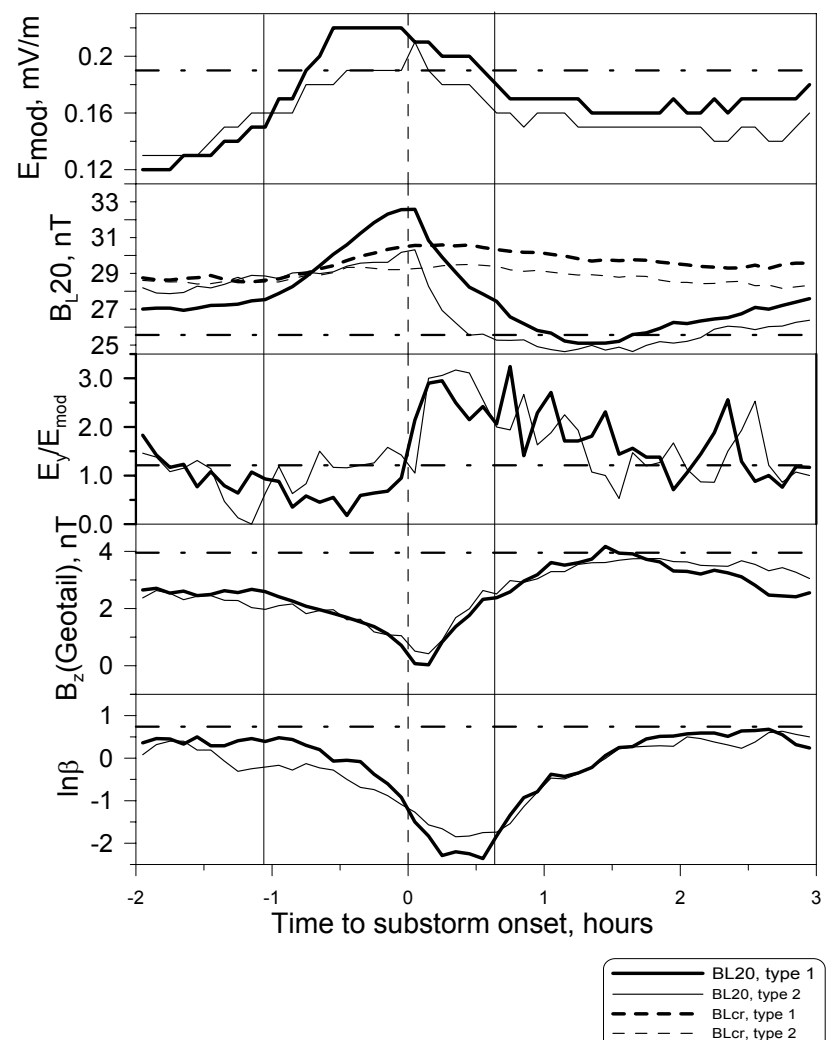

Fig. 3. Variation of the average values of the model electric field (top panel) and plasma sheet parameters for two types of substorms. The dash-dotted line indicates the average SMC values. Solid lines indicate the start of the growth phase (left) and recovery phase (righgt). The $\mathrm{B}_{L c r}$ was calculated based on IMF and solar wind data using the empirical formula (Eq. 5c in Shukhtina et al., 2004.)

growth was observed distinctly in 59 (type 1) events. In 46 events no (or very weak) BL growth (type 2) was observed, whereas in the remaining 40 events we could not separate the growth phase-related BL growth from that of the recovery phase of the preceding substorm. (We notice that in group 2 some $\mathrm{B}_{L}$ growth was seen during the hour preceding the onset, but it was less than $5 \%$ of its value at the beginning of the growth phase, as compared to $21 \%$ in type 1 events. Most of the changes were actually due to the variations of solar wind dynamic pressure in this data set, as revealed by $\mathrm{B}_{L c r}$ trace, also displayed in Fig. 3).

Therefore, in $1 / 3$ of all events (in type 2 events) the $\mathrm{B}_{L}$ behavior deviates from the classical scheme, and the reasons are interesting to explore. The two types of substorms are compared in Fig. 3, which reveals substantial differences between the two types of events, as well as some similarities. Both groups are characterized by an enhanced dayside transport rate $\mathrm{E}_{\text {mod }}$ but the plasma sheet flux transfer rates $\left(<\mathrm{E}_{y}>\right)$ are different. If averaged over $1 \mathrm{~h}$ duration of the growth phase, the $<\mathrm{E}_{y}>/<\mathrm{E}_{\bmod }>\sim 1.0$ during the growth phase of the type 2 substorms (means the transfer balance), whereas for the growth phase of the type 1 substorms this ratio is about 0.5 , indicating a significant surplus of the dayside-to-nightside flux transfer. 
Thus, different behavior of the plasma sheet convection in two groups gives a natural explanation of the different lobe magnetic flux variations. As for the other differences, type 1 events, as a rule, correspond to isolated substorms (starting on the quiet background), whereas the type 2 substorms usually develop on the disturbed background with strong convection.

Surprisingly, the weakness of the tail current growth in type 2 events does not imply the absence of the growth phase, as a global change preparing the magnetotail for the explosive energy dissipation. In fact, in both groups Fig. 3 shows the analogous behavior of other parameters, such as $\mathrm{B}_{z}$ depression (signature of the magnetic field stretching) and deep plasma $\beta$ decrease (implying severe plasma sheet thinning). These configurational changes rather than the tail current increase itself could be the principal factor leading to the tail explosion.

The enhanced intensity of the tail current is still a prerequisite for the expansion onset to occur. This is clear when comparing the average value of the lobe field $\mathrm{B}_{L}(20)$ at $\mathrm{T}=\mathrm{T}_{0}$ with its value computed based on solar wind conditions using the statistical relationship obtained for substorm onset epoch in Paper 1. The average values for both groups are similar and stay near their predicted levels. According to Paper 1 these critical levels $\mathrm{B}_{L}(20)_{c r}$ (shown by dashed lines on the $\mathrm{B}_{L}$ panel) are substantially higher as compared to the average $\mathrm{B}_{L}$ during the SMC events $(25.6 \mathrm{nT})$. In type 2 events the $\mathrm{B}_{L}$ trace stays near this predicted level for $1-2 \mathrm{~h}$ before the substorm onset. That means that the magnetotail was prepared for the explosion in terms of the free energy available, but it waited for the unstable configuration to launch the expansion phase.

Summarizing, we see that the convection regime during the growth phase of type 2 differs from the SMC regime (Fig. 2), as a thick plasma sheet with strongly closed magnetic flux characterizes the last one. During both types of convection nearly all magnetic flux from the dayside is reconnected on the nightside, however, the $\mathrm{B}_{L}$ values are different. Namely, the SMC $B_{L}$ value is lower but in contrast the type 2 growth phase $B_{L}$ is higher than the statistical average $B_{L}$ calculated by Fairfield (Fairfield, 1996).

\section{Concluding remarks}

In this statistical study we obtained the representative numerical values of the plasma sheet characteristics in the midtail during steady convection and two types of substorms. Based on the data obtained we emphasize the following major new results.

1. By averaging convection measurements over many events we found clear observational support for the magnetic flux imbalance paradigm by showing that:

(a) During steady convection the global rates of the magnetic flux transport to the tail from the dayside reconnection region and back are balanced, resulting in the stationary large-scale magnetic field in the magnetotail; (b) During substorms there exists a clear imbalance, with dayside-to-nightside flux transport prevailing over the return transport (by $\sim 40 \%$ on the average) during the growth phase, and inverse imbalance with nightside flux transport prevailing (by a factor of 2 on the average) during the expansion phase;

(c) A considerable part (one third) of all substorms analyzed showed very weak lobe field growth before the substorm onset. They were characterized by the enhanced plasma sheet convection and a balanced circulation in the magnetosphere.

2. In all substorms selected based on the signature of explosive lobe magnetic flux unloading (including those without $\mathrm{B}_{L}$ growth before onset) the magnetic field stretching and plasma sheet thinning, as well as the enhanced plasma sheet current have been observed. The variability of magnetotail behavior before the substorm onset indicates that the magnetosphere can probably reach the same critical state by passing through different dynamical paths.

3. The characteristics of the late substorm recovery phase are similar to those of the SMC state. It is consistent with the finding that all but one SMC event in our study were preceded by a substorm. The SMC regime seems to be a natural magnetospheric state following a substorm.

Acknowledgements. We thank the teams of Geotail and Wind spacecraft for the observations made available in the Internet throughout DARTS and CDAWeb data bases. We are also grateful to the teams of IMAGE, CANOPUS and 210 MM Internet sites. We thank WDC-C (Kyoto) for AE and SYM indices, O. Rassmussen for the Thule PC-index and O. A. Troshichev for providing 1-min PCindex from Vostok. This work was supported by the grants RFBR N04-05-64932a, the work of M. A. S. was supported by the Grant of Ministry of Education of Russian Federation E02-8.0-3.

Topical Editor T. Pulkkinen thanks a referee for his help in evaluating this paper.

\section{References}

Angelopoulos, V., Baumjohann, W., Kennel, C. F., Coroniti, F. V., Kivelson, M. G., Pellat, R., Walker, R. J., Lühr, H., and Paschmann, G.: Bursty bulk flows in the central plasma sheet, J. Geophys Res., 99, 10 967, 1992.

Baker, D. N., Pulkkinen, T. I., Hones Jr., E. W. , Belian, R. D. I., McPherron, R. L., Angelopulus, V.: Signatures of the substorm recovery phase at high-altitude spacecraft, J. Geophys Res., 99, 10967, 1994.

Bargatze, L. F., Baker, D. N., McPherron, R. L., Hones Jr., E. W.: Magnetospheric impulse response for many levels of geomagnetic activity, J. Geophys. Res., 90, 6387, 1985.

Baumjohann, W. G.: The near-Earth plasma sheet: an AMPTE/IRM perspective, Space Sci. Rev., 64 141, 1993.

Boyle, C. B., Reiff, P. H., and Harrison, M. R.: Empirical polar cap potentials, J. Geophys. Res., 102, 111, 1997.

Caan, M. N., McPherron, R. L., and Russell, C. T.: The statistical magnetic signatures of magnetospheric substorms, Planet. and Space Sci., 26, 2669, 1978.

Erickson, G. M.: A quasi-static magnetospheric convection model in two dimensions, J. Geophys. Res., 97, 6505, 1992. 
Foster, J. C., Fairfield, D. H., Ogilvie, K. W., and Rosenberg, T. J.: Relationship of interplanetary parameters and occurrence of magnetospheric substorms, J. Geophys. Res., 76, 6971, 1971.

Fairfield, D. H. and Jones, J.: Variability of the tail lobe field strength, J. Geophys. Res., 101, 7785, 1996.

Holzer, R. E., and Slavin, J. A.: A correlative study of magnetic flux transfer in the magnetosphere, J. Geophys. Res., 84, 2573, 1979.

Hsu, T.-S. and McPherron R. L.: The main onset of a magnetospheric substorm, in: Proceedings of Fourth International Conference on Substorms (ICS-4), Terra Scientific Publishing Company/Kluwer Academic Publishers, 79, 1998.

Lu, G., Reiff, P. H., Hairston, M. R., Heelis, R. A., and Karty, J. L.: Distribution of polar convection potential around the polar cap boundary as a function of the interplanetary magnetic field, J. Geophys. Res., 94, 13 447, 1989.

Lyons, L. R., Ruohoniemi, J. M., and Lu, G.: Substorm-associated changes in large-scale convection during the November, 24, 1996, Geospace environment modeling event, J. Geophys. Res., 106, 397, 2001.

Nakamura, R., Bargatze, L. F., Mukai, T., Nagai, T., Baker, K. B., Hairston, M. R., Reiff, P. H., Petrukovich, A. A., Nozdrachev, M., and Troshichev, O. A.: Response of the midtail electric field to enhanced solar wind energy input, J. Geophys. Res., 104, $17299,1999$.

Petrinec, S. M. and Russell, C. T.: Near-Earth magnetotail shape and size as determined from the magnetopause flaring angle, J. Geophys. Res., 101, 137, 1996.
Pulkkinen, T. I., Baker, D. N., Toivanen, P. K., Pellinen, R. J., Friedel, R. H. W., and Korth, A.: Magnetospheric field and current distribution during the substorm recovery phase, J. Geophys. Res., 99, 10 955, 1994.

Raeder, J., McPherron, R. L., Frank, L. A., Kokubun, S., Lu, G., Mukai, T., Paterson, W. R., Siggwarth, J. B., Singer, H. J., and Slavin, J. A.: Global simulation of the Geospace environement modeling substorm challenge event, J. Geophys. Res., 106, 381, 2001.

Reiff, P. H., Spiro, R. W., and Hill, T. W.: Dependence of polar cap potential drop on interplanetary parameters, J. Geophys. Res., 86, 7639, 1981.

Russell, C. T., and McPherron, R. L.: The magnetotail and substorms, Space Sci. Rev., 15, 205, 1973.

Rybal'chenko, V. V. and Sergeev, V. A.: Rate of magnetic flux buildup in the magnetospheric tail, Geomagn. Aeron., 25, 378, 1985.

Sergeev, V. A., Pellinen R. J., and Pulkkinen, T. I.: Steady magnetospheric convection: A review of recent results, Space Sci. Rev. 75, 551, 1996.

Sergeev, V. A., Pulkkinen, T. I., Pellinen, R. J., and Tsyganenko, N. A.: Hybrid state of the tail magnetic configuration during steady convection events, J. Geophys. Res., 99, 23 571, 1994.

Shukhtina, M. A., Dmitrieva, N. P., and Sergeev, V. A.: Quantitative magnetotail characteristic for different magnetospheric states, Ann. Geophysicae, 22, 1019, 2004.

Tsyganenko, N. A.: Effects of solar wind conditions on the global magnetospheric configuration as deduced from data-based field models, Rur. Space Agency Spec. Publ., ESA SP-389, 181, 1996. 